Vaccine in Seropositive Rheumatoid Arthritis Patients [abstract]. Arthritis Rheumatol. 2018; 70 (suppl 10).

Table 1. Characteristics of patients admitted for influenza compared to those admitted for other reasons

\begin{tabular}{lcc}
\hline Variable & $\begin{array}{c}\text { Not admission for influenza } \\
(\mathbf{n}=\mathbf{1 8 , 5 1 5 )}\end{array}$ & $\begin{array}{c}\text { Admission for influenza } \\
(\mathbf{n}=\mathbf{4 9 5})\end{array}$ \\
\hline Age (years) & $71.3 \pm 17.2$ & $74.1 \pm 13.7$ \\
$\%$ Male & $46.3 \%$ & $46.1 \%$ \\
Smoker & $3665(19.8 \%)$ & $126(25.4 \%)$ \\
RA & $700(3.8 \%)$ & $31(6.3 \%)$ \\
\hline
\end{tabular}

Disclosure of Interests: None declared

DOI: 10.1136/annrheumdis-2019-eular.7132

\section{FRI0059 SOLUBLE RECEPTOR FOR ADVANCED GLYCATION END PRODUCTS (SRAGE) AND RISK FOR CARDIOVASCULAR DISEASES IN FEMALES WITH RHEUMATOID ARTHRITIS}

Mitra Nadali ${ }^{1}$, Maria I. Bokarewa ${ }^{2}$, Sofia Töyrä Silfverswärd ${ }^{2}$, Malin Erlandsson ${ }^{2}$, Lovisa Lyngfelt ${ }^{2}$, Karin Me Andersson ${ }^{2}$, Rille Pullerits ${ }^{2} .{ }^{1}$ Institution of Medicine, Sahlgrenska Academy at University of Gothenburg, Department of Rheumatology and Inflammation Research, Gothenburg, Sweden; ${ }^{1}$ Institution of Medicine, Sahlgrenska Academy at University of Gothenburg, Department of Rheumatology and Inflammation Research, Gothenburg, Sweden

Background: Rheumatoid arthritis (RA) is strongly associated with increased frequency of cardiovascular disease (CVD), which remains the major cause of mortality in these patients. Current CVD risk assessment algorithms have limited predictive value for RA patients. Soluble receptor for advanced glycation end products (SRAGE) has recently emerged as a biomarker of inflammation with an inverse correlation with traditional CVD risk factors as age, hypertension and hypercholesterolemia. ${ }^{1}$

Objectives: In a cohort of female RA patients with no previous history of CVD, we assessed whether SRAGE levels were associated with increased risk of CVD during a prospective 5 years follow up.

Methods: Serum sRAGE levels were measured in 171 female RA patients (median age 53; range 21-71) at the inclusion to the study. The CVD risk was estimated using the Framingham algorithm and both traditional and RA associated risk factors for CVD were measured. All the patients were prospectively followed up to 5 years for new CV events, type II diabetes and medication for hypertension and hyperlipidemia. Statistical analysis was performed to compare CVD risk and actual events in the patients with low SRAGE $(<1700 \mathrm{pg} / \mathrm{ml})$.

Results: The comparison of patients with low sRAGE $(n=125)$ and normal-high sRAGE $(n=46)$ found no significant differences in frequency of the traditional CVD risk factors including age>60 years (30\% vs $41 \%)$, overweight $(50 \%$ vs $41 \%)$, smoking (14\% vs $11 \%$ ), incidental hypertension $(16 \%$ vs $16 \%)$ and hypercholesterolemia $(56 \%$ vs $67 \%)$ at baseline and led to similar estimated CVD risk $(7.65 \%$ vs $8,45 \%)$. The RA-related CVD risk factors including disease duration >10years (37\% vs $39 \%$ ), presence of autoantibodies ( $89 \%$ vs $95 \%$ ), disease activity (DAS28, $46 \%$ vs $60 \%$ ) were also similar in the low-and high sRAGE groups. At 5 years follow up, 11 new CVD events were registered. The events occurred with similar frequency in the low sRAGE and high sRAGE groups $(5.6 \%$ vs $8.7 \%)$. Despite a lack of difference in CVD events, we did observe a significant increase in frequency of new medication for hypertension in the low sRAGE group $(21.5 \%$ vs. $63 \%, p=0.01)$, but not in medication for type II diabetes or statins.

Conclusion: In this study, we found no association between serum levels of SRAGE and the estimated CVD risk or actually occurred CVD events in female RA patients.

\section{REFERENCE:}

[1] Santilli, F., et al. (2009). "Soluble forms of RAGE in human diseases: clinical and therapeutical implications." Curr Med Chem 16(8): 940-952.

Disclosure of Interests: None declared

DOI: 10.1136/annrheumdis-2019-eular.6236

\section{FRI0060 \\ NEUROCOGNITIVE PROFILE IN CHILDREN BORN TO MOTHERS WITH CHRONIC ARTHRITIS: WHICH RELATIONSHIP WITH MATERNAL FEELING OF DISEASE?}

Cecilia Nalli ${ }^{1}$, Jessica Galli ${ }^{2}$, Angela Merlini ${ }^{2}$, Chiara Asperti ${ }^{1}$, Laura Andreoli ${ }^{1}$, Elisa Fazzi" ${ }^{2}$, Angela Tincani ${ }^{1}$. 'Unit of Rheumatology and Clinical Immunology, Spedali Civili and University of Brescia, Brescia, Italy; ${ }^{2}$ Unit of Child Neurology and Psychiatry, Spedali Civili and University of Brescia, Brescia, Italy

Background: Chronic Arthritis could begin in women in childbearing age with several consequent complications in family planning. There are few data on long term outcome of children born from these mothers.

Objectives: Evaluate the neurocognitive profile of children born to mothers with Rheumatoid Arthritis (RA), Psoriasic Arthritis (PsA), Ankylosing Spondylitis (SpA) and studied the psychological outcome of these patients in relationship to their pregnancies.

Methods: We evaluated 16 women (11 RA, 3 PsA, 2 SpA) with diagnosis before pregnancy and school-age children. Information about maternal disease and treatment during pregnancy were collected from medical records. The work was performed by a multidisciplinary team (rheumatologists and pediatric neurologists). The Child Behavior Check List (CBCL 618), a home-made questionnaire and others validate questionnaires (STATE-TRAIT ANXIETY INVENTORY - STAI, EDIMBURGH POSTNATAL DEPRESSION SCALE - EPDS, PAYKEL scale of stressful life events) were submitted to all mothers. A neurological physical exam was performed by a pediatric neurologist in all children. Intellectual functioning were assessed by Wechsler scale for corrected age. We also applied standardized batteries to evaluate academic learning. The study was approved by the local ethical committee.

Results: We evaluated 18 children ( $F / M=1 / 1$, median age 7,4 years, range 6.4-14). All children were born at term and with normal weight The neurological physical exam and the intelligence level were normal in all children. One child displayed a specific learning disability in reading activities (10\% vs $2,5-3,5 \%$ of general pediatric population). We found in 6 children (33\%) behavior aspects of somatization and anxiety/ depression (internalizing problem), in $2(11 \%)$ externalizing problems (oppositional-provocative behavior) and in 5 (28\%) an "adult profile". From maternal evaluations we found significant correlations between activity of disease during pregnancy and difficult caregiving and breastfeeding ( $p=0,005$ and $p=0,0128$ respectively). Finally, a relationship also exists between maternal depression/anxiety and need of psychological support.

Conclusion: This preliminary study on children born to mothers with chronic arthritis showed in children a prevalent pattern of anxiety/depression and an adult, mature profile, expression of insecurity. Therefore, data from $C B C L$ showed that these children were less involved in sports or playful activities than peers and were more involved in school activities. It seemed they would invest more energies in intellectual functioning, as noted in children affected by chronic diseases such as asthma or juvenile idiopathic arthritis. On the maternal side clearly emerged a diffuse state of anxiety, accentuated by having an active disease during pregnancy and puerperium. Postpartum depression was detected in these mothers with higher levels than general population (39\% vs $10-15 \%$ ): it could be desiderable to give these mothers a psychological support during this period. The practical message of our study is also that children born to women with chronic arthritis may need a long-term followup in order to detect and correct any neurological, behavioral or cognitive alteration as early as possible.

\section{REFERENCE:}

[1] Bomba M, Galli J, Nacinovich R, et al. Neuropsychiatric aid in children born to patients with rheumatic diseases. Clin Exp Rheumatol. 2010 SepOct;28(5):767-73.

Disclosure of Interests: Cecilia Nalli: None declared, Jessica Galli: None declared, Angela Merlini: None declared, Chiara Asperti: None declared, Laura Andreoli: None declared, Elisa Fazzi: None declared, Angela Tincani Consultant for: UCB, Pfizer, Abbvie, BMS, Sanofi, Roche, GSK, AlphaSigma, Lilly, Jannsen, Cellgene, Novartis DOI: 10.1136/annrheumdis-2019-eular.2644 